\title{
Some fundamental relations in the projective differential geometry of ruled surfaces ${ }^{(*)}$.
}

\author{
(By A. F. Cahpenter, Seattle, Wash, U. S. A.)
}

\section{INTRODUGTI ON.}

In his projective theory of ruled surfaces $\left(^{* *}\right)$ WiLczYnskr makes frequent use of a moving tetrahedron of reference $P_{y} P_{z} P_{p} P_{\sigma}$, two of whose vertices $P_{y}$ and $P_{z}$ lie on a generator $L_{y z}$ of the surface and the remaining two of which are semi-covariantly related to the first pair $(* * *)$.

The homogeneous coordinates $\left(y_{1}, \ldots, y_{4}\right)$ and $\left(z_{1}, \ldots, z_{4}\right)$ of $P_{y}$ and $P_{z}$ constitute a fundamental system of simultaneous solutions of the pair of equations

$$
\left.\begin{array}{l}
y^{\prime \prime}+p_{11} y^{\prime}+p_{12} z^{\prime}+q_{11} y+q_{12} z=0, \\
z^{\prime \prime}+p_{21} y^{\prime}+p_{22} z^{\prime}+q_{21} y+q_{22} z=0,
\end{array}\right\}
$$

upon which this theory is based, while $\left(\rho_{1}, \ldots, p_{4}\right)$ and $\left(\sigma_{1}, \ldots, \sigma_{4}\right)$, the coordinates of the vertices $P_{\rho}$ and $P_{\sigma}$ are given by the corresponding values

$$
\rho_{k}=2 y_{k}^{\prime}+p_{11} y_{k}+p_{12} z_{k}, \quad \sigma_{k}=2 z_{k}^{\prime}+p_{31} y_{k}+p_{22} z_{k}, \quad(k=1, \ldots, 4)
$$

of the semi-covariants $\rho$ and $\sigma\left({ }^{* * * *}\right)$.

(*) Presented to the American Mathematical Society, April 1915 and November 1916.

(**) WiLczynski, Projective Differential Geometry of Curves and Ruled Surfaces. Hereafter designated W.

(***) The vertices $P_{y}, P_{z}, P_{\varrho}, P_{\sigma}$ all lie on the quadric which osculates the ruled surface along $I_{y z}$ while the lines $P_{y} P_{z}, P_{\varrho} P_{\sigma}$ are generators of one regulus, and $P_{y} P_{\varrho}, P_{z} P_{\sigma}$ generators of the other regulus, of this quadric. W., p. 147.

$\left(*_{* * *}\right) \rho$ and $\sigma$ are not in fact semi-covariants altho for convenience and following WrcczYNSKI we shall speak of them as such. They are quantities which are cogredient with $y$ and $z$. See W., p. 124. 
The coordinates $y_{k}, z_{k}, \rho_{k}, \sigma_{k}$ are functions of the independent variable $x$ of system (A), so that $x$ figures as the parameter of the surface. The tetrahedron of reference varies with the parameter.

It is the purpose of this paper (I) to determine the conditions satisfied by the coordinates of points, planes and lines which are fixed in space, and (II) to indicate by. a number of examples the value of the above conditions in deducing further theorems on ruled surfaces.

\section{Gonditions for Fixed Points, Planes and Lines.}

Let $P$ be a point whose coordinates with respect to the moving tetrahedron are $(\alpha, \beta, \gamma, \delta)$. Then the expression $\left({ }^{*}\right)$

$$
p=\alpha y+\beta z+\gamma \beta+\delta \sigma,
$$

will represent the point $P$ when referred to a fixed tetrahedron. We have, by making use of equations (1)

$$
p=\left(\alpha+p_{11} \gamma+p_{21} \delta\right) y+\left(\beta+p_{12} \gamma+p_{22} \delta\right) z+2 \gamma y^{\prime}+2 \delta z^{\prime}
$$

If $P$ is a fixed point, a change $\delta x$ in $x$ producing a change $\delta p$ in $p$ must be such that

$$
p+\delta p \equiv(1+\theta \delta x) p
$$

where $\theta$ may be an arbitrary function of $x$. We must therefore have

$$
p^{\prime} \equiv \theta p, \quad\left(y, z, y^{\prime}, z^{\prime}\right) .
$$

If we express both members of (4) in terms of $y, z, y^{\prime}, z^{\prime}$, and equate corresponding coefficients, we find

$$
\begin{gathered}
\alpha^{\prime}+p_{11} \gamma^{\prime}+p_{21} \delta^{\prime}-\theta \alpha+\left(p_{11}^{\prime}-2 q_{11}-p_{11} \theta\right) \gamma+\left(p_{21}^{\prime}-2 q_{21}-p_{21} \theta\right) \delta=0 \\
\beta^{\prime}+p_{12} \gamma^{\prime}+p_{22} \delta^{\prime}-\theta \beta+\left(p_{12}^{\prime}-2 q_{12}-p_{12} \theta\right) \gamma+\left(p_{22}^{\prime}-2 q_{22}-p_{22} \theta\right) \delta=0 \\
2 \gamma^{\prime}+\alpha-\left(2 \theta+p_{11}\right) \gamma-p_{21} \delta=0,2 \delta^{\prime}+\beta-p_{12} \gamma-\left(2 \theta+p_{22}\right) \delta=0
\end{gathered}
$$

(*) W., p. 191 . 
or

where

$$
\begin{aligned}
& 2 \alpha^{\prime}=\left(2 \theta+p_{11}\right) \alpha+p_{21} \beta-u_{11} \gamma-u_{21} \delta \\
& 2 \beta^{\prime}=p_{12} \alpha+\left(2 \theta+p_{22}\right) \beta-u_{12} \gamma-u_{22} \delta \\
& 2 \gamma^{\prime}=-\alpha+\left(2 \theta+p_{1 i}\right) \gamma+p_{21} \delta \\
& 2 \delta^{\prime}=-\beta+p_{12} \gamma+\left(2 \theta+p_{22}\right) \delta
\end{aligned}
$$

$$
\left.\begin{array}{c}
u_{11}=2 p_{11}^{\prime}-4 q_{11}+p_{11}^{2}+p_{12} p_{21}, \\
u_{12}=2 p_{12}^{\prime}-4 q_{12}+p_{12}\left(p_{11}+p_{22}\right), \\
u_{21}=2 p_{21}^{\prime}-4 q_{21}+p_{21}\left(p_{11}+p_{22}\right), \\
u_{22}=2 p_{22}^{\prime}-4 q_{22}+p_{22}^{2}+p_{12} p_{21} .
\end{array}\right\}
$$

Equations (5) express the conditions which must be satisfied by the coordinates $(\alpha, \beta, \gamma, \delta)$ of a point if this point is to be fixed in space. Moreover conditions (5) imply equation (4). Conditions (5) are therefore both necessary and sufficient to make $P$ a fixed point.

By eliminating $\alpha$ and $\beta$ from (5) there are obtained two relations involving $\gamma, \delta$ and their first and second derivatives, of the form

$$
\begin{gathered}
\delta^{\prime \prime}-\left(2 \theta+p_{22}\right) \delta^{\prime}-p_{12} \gamma^{\prime}+\left(\theta^{2}+p_{22} \theta-\theta^{\prime}-p_{22}^{\prime}+q_{22}\right) \delta+ \\
+\left(p_{12} \theta-p_{12}^{\prime}+q_{12}\right) \gamma=0 \\
\gamma^{\prime \prime}-p_{21} \delta^{\prime}-\left(2 \theta+p_{11}\right) \gamma^{\prime}+\left(p_{21} \theta-p_{21}^{\prime}+q_{21}\right) \delta+ \\
+\left(\theta^{2}+p_{11} \theta-\theta^{\prime}-p_{11}^{\prime}+q_{11}\right) \gamma=0 .
\end{gathered}
$$

Since $\theta$ may be an arbitrary function of $x$, we may put $\theta=-1 / 2\left(p_{11}+p_{22}\right)$.

Equations (7) then reduce to

$$
\left.\begin{array}{l}
\delta^{\prime \prime}+p_{11} \delta^{\prime}-p_{12} \gamma^{\prime}+\left[q_{11}+1 / 1\left(u_{11}-u_{22}\right)\right] \delta-\left(q_{12}+1 / 2 u_{12}\right) \gamma=0, \\
\gamma^{\prime \prime}-p_{21} \delta^{\prime}+p_{22} \gamma^{\prime}-\left(q_{21}+1 / 2 u_{21}\right) \delta+\left[q_{22}+1 / 4\left(u_{22}-u_{11}\right)\right] \gamma=0 .
\end{array}\right\}
$$

Now with every system of form (A) there is associated an adjoint system. This is the system satisfied by the coordinates of the planes tangent to the ruled surface $S$ at the points $P_{y}$ and $P_{z}$. The adjoint system may also be interpreted as belonging to a ruled surface $\Sigma$ dualistic to $S\left(^{*}\right)$. The system

(*) W., Ch. V, $\$ 83,4,5$. 
adjoined to $(\mathrm{A})$ is

$$
\left.\begin{array}{l}
U^{\prime \prime}+p_{11} U^{\prime}+p_{12} V^{\prime}+\left[q_{11}+1 / 4\left(u_{11}-u_{22}\right)\right] U+\left(q_{12}+1 / 2 u_{12}\right) V=0, \\
V^{\prime \prime}+p_{21} U^{\prime}+p_{22} V^{\prime}+\left(q_{21}+1 / 2 u_{21}\right) U+\left[q_{22}+1 / 4\left(u_{22}-u_{11}\right)\right] V=0 .
\end{array}\right\}
$$

Comparison of (8) with (9) shows that $(\delta,-\gamma)$ constitute a pair of simultaneous solutions of system (9). Moreover if we form for system (8) the semi-covariants corresponding to $\rho$ and $\sigma$ of equations (1), we. find by $\left(5_{3}\right)$ and $\left(5_{4}\right)$ that $(-\beta, \alpha)$ are exactly these semi-covariants. Whence, if the expression $\alpha y+\beta z+\gamma \rho+\delta \sigma$ represents a point $P$ fixed in space then $\delta$ and $-\gamma$ will constitute $"$ pair of simultaneous solutions of the system of differential equations adjoined to the system which defines the ruled surface $S$, and $-\beta$ and $\alpha$ will be the values assumed respectively by the semi-covariants $p$ and $\sigma$ of this system.

It is also of interest to note that $(\delta,-\gamma,-\beta, \alpha)$ are the coordinates of the polar plane of the point $(\alpha, \beta, \gamma, \delta)$ taken with respect to the quadric $x_{1} x_{4}-x_{2} x_{3}=0$ which osculates the surface $S$ along the generator $L_{y z}\left({ }^{*}\right)$.

We now consider three points $P_{1}, P_{2}, P_{3}$ whose coordinates $\left(\alpha_{1}, \beta_{1}\right.$, $\left.\gamma_{1}, \delta_{1}\right),\left(\alpha_{2}, \beta_{2}, \gamma_{2}, \delta_{2}\right),\left(\alpha_{3}, \beta_{3}, \gamma_{3}, \delta_{3}\right)$ satisfy conditions (5). The plane $n$ determined by these three points is then fixed in space. Let the coordinates of $\Pi$ be $(k, \lambda, \mu, \nu)$. They are proportional to the four determinants $\left(\beta_{1} \gamma_{2} \delta_{3}\right)$, $-\left(\alpha_{1} \gamma_{2} \delta_{3}\right),\left(\alpha_{1} \beta_{2} \delta_{3}\right)$ and $-\left(\alpha_{1} \beta_{2} \gamma_{3}\right)$. Let

$$
k=\left(\beta_{1} \gamma_{2} \delta_{3}\right) \varphi, \quad \lambda=-\left(\alpha_{1} \gamma_{2} \delta_{3}\right) \varphi, \quad \mu=\left(\alpha_{1} \beta_{2} \delta_{3}\right) \varphi, \quad \nu=-\left(\alpha_{1} \beta_{2} \gamma_{3}\right) \varphi,
$$

where $\varphi$ is an arbitrary function $\equiv \equiv 0$. Then we find by differentiating and making use of (5) that

$$
\left.\begin{array}{l}
2 k^{\prime}=\left(n+p_{11}+2 p_{22}\right) k-p_{12} \lambda+\mu, \\
2 \lambda^{\prime}=-p_{21} k+\left(n+2 p_{11}+p_{22}\right) \lambda+\nu, \\
2 \mu^{\prime}=u_{11} k+u_{12} \lambda+\left(n+p_{11}+2 p_{22}\right) u-p_{12} \nu, \\
2 \nu^{\prime}=u_{21} k+u_{22} \lambda-p_{21} \mu+\left(n+2 p_{11}+p_{22}\right) \nu,
\end{array}\right\}
$$

where $n=6 \theta+2 \varphi^{\prime} / \varphi$. Equations (10) express the conditions which must be satisfied by the coordinates $(k, \lambda, \mu, \nu)$ of a plane which is fixed in space. But these conditions are likewise sufficient. For let us think of the generator $L_{y z}$

(*) W., p. 191. 
of $S$ as being determined by the intersection of the two planes tangent to $S$ at $P_{y}$ and $P_{z}$. Their coordinates $\left(U_{1}, \ldots, U_{4}\right)$ and $\left(V_{1}, \ldots, V_{4}\right)$ constitute a fundamental system of simultaneous solutions of system (9). The tetrahedron of reference associated with system (9) will be identical with that already used, each vertex being replaced by that one of the faces thru it which is at the same time tangent to the osculating quadric at that vertex $\left(^{*}\right)$. With respect to this tetrahedron the expression for any plane $\Pi$ whose coordinates are $(k, \lambda, \mu, \nu)$ will be

$$
\pi=k S-\lambda R-\mu \cdot V+\nu U
$$

where

$$
R=2 U^{\prime}+p_{11} U+p_{12} V, \quad S=2 V^{\prime}+p_{21} U+p_{22} V
$$

are the semi-covariants formed for system (9) just as $\rho$ and $\sigma$ are formed for system (A). Differentiating (11) and making use of (9) and (12) we have, after imposing conditions (10)

$$
2 \pi^{\prime}=\left(n+p_{11}+p_{22}\right)(k S-\lambda R-\mu V+\nu V)=\left(n+p_{11}+p_{22}\right) \pi,
$$

that is, the plane $\Pi$ is fixed in space.

By eliminating $\mu$ and $\nu$ from (10) we obtain two relations involving $k, \lambda$ and their first and second derivatives of the form

$$
\left.\begin{array}{r}
k^{\prime \prime}-\left(n+p_{11}+2 p_{22}\right) k^{\prime}+p_{12} \lambda^{\prime}+ \\
+\left[1_{4} n^{2}-1 / 2 n^{\prime}+1 / 2\left(p_{11}+2 p_{22}\right) n-p_{11}^{\prime}-p_{22}^{\prime}+p_{11} p_{22}+p_{22}^{2}+q_{11}\right] k- \\
\quad-\left({ }^{1} / 2 p_{12} n+p_{11} p_{12}+p_{12} p_{22}-q_{12}\right) \lambda=0, \\
\lambda^{\prime \prime}+p_{21} k^{\prime}-\left(n+2 p_{11}+p_{22}\right) \lambda^{\prime}- \\
-\left({ }^{1} / 2 p_{21} n+p_{11} p_{21}+p_{21} p_{22}-q_{21}\right) k+ \\
+\left[{ }^{1} / 4 n^{2}-1 / 2 n^{\prime}+1 / 2\left(2 p_{11}+p_{22}\right) n-p_{11}^{\prime}-p_{22}^{\prime}+p_{11} p_{22}+p_{11}^{2}+q_{22}\right] \lambda=0 .
\end{array}\right\}
$$

When $\theta=0$ and $\varphi=e^{-\int\left(p_{11}+p_{22}\right) d x}$, equations (14) become precisely those of system (A) with $k$ and $\lambda$ replacing $y$ and $z$ respectively. Moreover for these values of $\theta$ and $\varphi, \mu$ and $\nu$ replace the semi-covariants $\rho$ and $\sigma$ as may be seen by comparing (1) with $\left(10_{1}\right)$ and $\left(10_{2}\right)$. Whence if the expression $k S-\lambda R-\mu V+\nu U$ represents a plane II fixed in space, then $k$ and $\lambda$ will

$\left(^{*}\right)$ See foot note, page 1 . 
constitute a pair of simultaneous solutions of the system of differential equations defining the ruled surface $S$ and $\mu$ and $\nu$ will be the values assumed respectively by the semi-covariants $p$ and of this system.

The conditions for a fixed line can be obtained in an analogous manner. Consider two points $P_{1}$ and $P_{2}$ whose coordinates $\left(\alpha_{1}, \beta_{1}, \gamma_{1}, \delta_{1}\right)$ and $\left(\alpha_{2}, \beta_{2}, \gamma_{2}, \delta_{2}\right)$ satisfy conditions (5). The fixed line determined by these two points will then have for its Plückerian coordinates the expressions

$$
\begin{aligned}
& \omega_{12}=\left(\alpha_{1} \beta_{2}-\alpha_{2} \beta_{1}\right) \psi, \quad \omega_{13}=\left(\alpha_{1} \gamma_{2}-\alpha_{2} \gamma_{1}\right) \psi, \quad \omega_{14}=\left(\alpha_{1} \delta_{2}-\alpha_{2} \delta_{1}\right) \psi \\
& \omega_{23}=\left(\beta_{1} \gamma_{2}-\beta_{2} \gamma_{1}\right) \psi, \quad \omega_{42}=\left(\delta_{1} \beta_{2}-\delta_{2} \beta_{1}\right) \psi, \quad \omega_{34}=\left(\gamma_{1} \delta_{2}-\gamma_{2} \delta_{1}\right) \psi,
\end{aligned}
$$

where $\psi$ is an arbitrary function $\equiv \equiv 0$. By differentiating (15) and making use of (5) we obtain

$$
\begin{aligned}
& 2 \omega_{12}^{\prime}=\left(\zeta+p_{11}+p_{22}\right) \omega_{12}-u_{12} \omega_{13}-u_{22} \omega_{14}+u_{11} \omega_{23}-u_{21} \omega_{42} \\
& 2 \omega_{13}^{\prime}=\left(\zeta+2 p_{11}\right) \omega_{18}+p_{21}\left(\omega_{14}+\omega_{23}\right)+u_{21} \omega_{34}, \\
& 2 \omega_{14}^{\prime}=-\omega_{12}+p_{12} \omega_{13}+\left(\zeta+p_{11}+p_{22}\right) \omega_{14}-p_{21} \omega_{42}-u_{11} \omega_{34}, \\
& 2 \omega_{23}^{\prime}=\omega_{12}+p_{12} \omega_{13}+\left(\zeta+p_{11}+p_{22}\right) \omega_{23}-p_{21} \omega_{12}+u_{22} \omega_{34}, \\
& 2 \omega_{42}^{\prime}=-p_{12}\left(\omega_{14}+\omega_{23}\right)+\left(\zeta+2 p_{22}\right) \omega_{42}+u_{12} \omega_{34}, \\
& 2 \omega_{34}^{\prime}=-\omega_{14}+\omega_{23}+\left(\zeta+p_{11}+p_{22}\right) \omega_{34},
\end{aligned}
$$

where $\zeta=4 \theta+2 \psi^{\prime} / \psi$. These are the conditions which must be satisfied by the coordinates of a fixed line. Moreover if the coordinates of any line $l$ satisfy conditions (16) the line is fixed. For let $\left(\alpha_{1}, \beta_{1}, \gamma_{1}, \delta_{1}\right)$ be the coordinates of any fixed point whatever. Then the coordinates of the plane II determined by this point and the line $l$ are

$$
\left.\begin{array}{ll}
k=\beta \omega_{34}+\gamma \omega_{42}+\delta \omega_{23}, & \lambda=-\alpha \omega_{34}+\gamma \omega_{14}-\delta \omega_{13}, \\
\mu=-\alpha \omega_{42}-\beta \omega_{14}+\delta \omega_{12}, & \nu=-\alpha \omega_{23}+\beta \omega_{13}-\gamma \omega_{12} .
\end{array}\right\}
$$

By differentiating (17) and imposing conditions (16) and (5) we find

$$
\begin{aligned}
& 2 k^{\prime}=\left(\xi+p_{11}+2 p_{22}\right) k-p_{12} \lambda+\mu, \\
& 2 \lambda^{\prime}=-p_{21} k+\left(\xi+2 p_{11}+p_{22}\right) \lambda+\nu, \\
& 2 a^{\prime}=u_{11} k+u_{12} \lambda+\left(\xi+p_{11}+2 p_{22}\right) \mu-p_{12} \nu, \\
& 2 v^{\prime}=u_{21} k+u_{22} \lambda-p_{21} \mu+\left(\xi+2 p_{11}+p_{22}\right) \nu,
\end{aligned}
$$

where $\xi=6 \theta+2 \psi^{\prime} / \psi$. But equations (18) are identical with equations (10) since $\psi$ and $\varphi$ are subject to the same conditions. Whence $\Pi$ is a fixed plane 
and therefore $l$ is a fixed line. It follows that for a line to be fixed in space it is necessary and sufficient that its Plückerian coordinates satisfy conditions (16).

Now the six coordinates of the line $L_{y z}$ satisfy a linear homogeneous differential equation of the sixth order $\left(^{*}\right)$. If system (A) be taken in the semi-canonical form, that is

$$
y^{\prime \prime}+q_{11} y+q_{12} z=0, \quad z^{\prime \prime}+q_{21} y+q_{82} z=0,
$$

this sixth order differential equation can be written in the form

where

$$
\left|\begin{array}{lllll}
s & q^{\prime \prime \prime}{ }_{11} & q^{\prime \prime \prime}{ }_{12} & q^{\prime \prime \prime}{ }_{21} & q^{\prime \prime \prime}{ }_{22} \\
t & q^{\prime \prime}{ }_{11} & q^{\prime \prime}{ }_{12} & q_{21}^{\prime \prime} & q^{\prime \prime}{ }_{22} \\
w & q_{11}^{\prime} & q_{12}^{\prime} & q_{21}^{\prime} & q_{22}^{\prime} \\
v^{\prime} & q_{11} & q_{12} & q_{21} & q_{22} \\
\omega^{\prime} & -1 & 0 & 0 & -1
\end{array}\right|=0,
$$

$$
\begin{aligned}
2 v & =\omega^{\prime \prime}+\left(q_{11}+q_{22}\right) \omega \\
w & =v^{\prime \prime}-2\left(q_{11} q_{22}-q_{12} q_{21}\right) \omega+\left(q_{11}+q_{22}\right) v \\
t & =w^{\prime}-\left(q_{11} q_{22}^{\prime}+q_{11}^{\prime} q_{22}-q_{12} q_{21}^{\prime}-q_{12}^{\prime} q_{21}\right) \omega+\left(q_{11}^{\prime}+q_{22}^{\prime}\right) v \\
s & =t^{\prime}-\left(q_{11} q_{22}^{\prime \prime}+q_{11}^{\prime \prime} q_{22}-q_{12} q_{21}^{\prime \prime}-q_{12}^{\prime \prime} q_{21}\right) \omega+\left(q_{11}^{\prime \prime}+q_{22}^{\prime \prime}\right) v .
\end{aligned}
$$

For this semi-canonical form of (A), and with $\theta=0$ and $\psi=$ constant, equations (16) become

$$
\begin{gathered}
\omega_{12}^{\prime}=2 q_{18} \omega_{13}+2 q_{22} \omega_{14}-2 q_{11} \omega_{93}+2 q_{21} \omega_{42}, \\
\omega_{13}^{\prime}=-2 q_{21} \omega_{34}, \quad 2 \omega_{14}^{\prime}=-\omega_{18}+4 q_{11} \omega_{34}, \quad 2 \omega_{23}^{\prime}=\omega_{19}-4 q_{22} \omega_{34.5} \\
\omega_{42}^{\prime}=-2 q_{12} \omega_{34}, \quad 2 \omega_{34}^{\prime}=-\omega_{14}+\omega_{23} .
\end{gathered}
$$

From $\left(21_{3}\right),\left(21_{4}\right)$ and $\left(21_{6}\right)$ we find

$$
\omega_{12}=2 v=2 \omega^{\prime \prime}{ }_{34}+2\left(q_{11}+q_{22}\right) \omega_{34} ;
$$

from $\left(21_{1}\right)$ and (22)

$$
v^{\prime}=-q_{11} \omega_{28}+q_{12} \omega_{13}+q_{21} \omega_{12}+q_{22} \omega_{14} ;
$$

(*) W., pp. 165, 166. 
from (21), (22) and (23)

$$
\left.\begin{array}{rl}
v & =v^{\prime \prime}-4\left(q_{11} q_{22}-q_{12} q_{21}\right) \omega_{34}+\left(q_{11}+q_{22}\right) v= \\
& =-q_{: 1}^{\prime} \omega_{23}+q_{12}^{\prime} \omega_{13}+q_{21}^{\prime} \omega_{42}+q_{22}^{\prime} \omega_{14} .
\end{array}\right\}
$$

Similarly we get

$$
\left.\begin{array}{rl}
t & =w^{\prime}-2\left(q_{11} q_{22}^{\prime}+q_{11}^{\prime} q_{22}-q_{12} q_{21}^{\prime}-q_{12}^{\prime} q_{21}\right) \omega_{34}+\left(q_{11}^{\prime}+q_{22}^{\prime}\right) v= \\
& =-q_{11}^{\prime \prime} \omega_{23}+q_{12}^{\prime \prime} \omega_{13}+q_{21}^{\prime \prime} \omega_{42}+q_{22}^{\prime \prime} \omega_{14}
\end{array}\right\}
$$

and

$$
\left.\begin{array}{rl}
s & =t^{\prime}-2\left(q_{11} q_{22}^{\prime \prime}+q_{11}^{\prime \prime} q_{22}-q_{12} q_{21}^{\prime \prime}-q_{12}^{\prime \prime} q_{21}\right) \omega_{34}+\left(q_{11}^{\prime \prime}+q_{22}^{\prime \prime}\right) v= \\
& =-q^{\prime \prime \prime}{ }_{1 !} \omega_{23}+q_{12}^{\prime \prime \prime} \omega_{13}+q_{21}^{\prime \prime \prime} \omega_{42}+q_{22}^{\prime \prime \prime} \omega_{14} .
\end{array}\right\}
$$

Eliminating $\omega_{23}, \omega_{13}^{\frac{3}{3}}, \omega_{42}$ and $\omega_{14}$ from $\left(21_{6}\right),(23),(24),(25)$ and (26) gives

$$
\left|\begin{array}{ccccc}
s & q^{\prime \prime \prime}{ }_{11} & q^{\prime \prime \prime}{ }_{12} & q^{\prime \prime \prime}{ }_{21} & q^{\prime \prime \prime}{ }_{22} \\
t & q^{\prime \prime}{ }_{11}^{\prime} & q_{12}^{\prime \prime} & q_{21}^{\prime \prime} & q_{22}^{\prime \prime} \\
w & q_{11}^{\prime} & q_{12}^{\prime} & q_{21}^{\prime} & q_{22}^{\prime} \\
v^{\prime} & q_{11} & q_{12} & q_{21} & q_{22} \\
2 \omega_{34}^{\prime} & --1 & 0 & 0 & -1
\end{array}\right|=0 .
$$

A comparison of (22)-(27) with (19) and (20) shows that $2 \omega_{34}$, and therefore $\omega_{34}$, is a solution of the differential equation of the sixth order satisfied by the line coordinates of the generators of the ruled surface $S$.

\section{A Number of Theorems on Ruled Surfaces.}

In (5), (10) and (16) are given conditions on the coordinates of points, planes and lines necessary and sufficient to make these elements fixed in space when the system of coordinates employed is that of the moving tetrahedron $P_{y} P_{z} P_{\rho} P_{\sigma}$. We proceed to the proofs of a number of theorems based upon these conditions.

Let $P_{k}$ with coordinates $\left(\alpha_{k}, \beta_{k}, \gamma_{k}, \delta_{k}\right),(k=1, \ldots, 4)$ be four points forming a non-degenerate tetrahedron fixed in space. Then for each set of 
four coordinates conditions (5) and (8) must hold. Moreover the determinant

$$
\left|\begin{array}{llll}
\alpha_{1} & \alpha_{2} & \alpha_{3} & \alpha_{4} \\
\beta_{1} & \beta_{2} & \beta_{3} & \beta_{4} \\
\gamma_{1} & \gamma_{2} & \gamma_{3} & \gamma_{4} \\
\delta_{1} & \delta_{2} & \delta_{3} & \delta_{4}
\end{array}\right|=0
$$

By means of $\left(5_{3}\right)$ and $\left(5_{4}\right)$ condition (28) becomes, except for a constant factor

$$
\left|\begin{array}{rrrr}
-\gamma_{1}^{\prime} & -\gamma_{2}^{\prime} & -\gamma_{3}^{\prime} & -\gamma_{4}^{\prime} \\
\delta_{1}^{\prime} & \delta_{2}^{\prime} & \delta_{3}^{\prime} & \delta^{\prime}{ }_{4} \\
-\gamma_{1} & -\gamma_{2} & -\gamma_{3} & -\gamma_{4} \\
\delta_{1} & \delta_{2} & \delta_{3} & \delta_{4}
\end{array}\right|==0 .
$$

But (29), in view of (7), implies that the two sets of four functions $\left(\delta_{1}, \ldots, \delta_{4}\right)$ and $\left(-\gamma_{1}, \ldots,-\gamma_{1}\right)$ are proportional to a fundamental system of simultaneous solutions of system (9), the adjoint of system (A), and further that $\left(-\beta_{1}, \ldots,-\beta_{4}\right)$ and $\left(\alpha_{1}, \ldots, \alpha_{4}\right)$ are the values assumed respectively by the semi-covariants $R_{k}$ and $S_{k},(k=1, \ldots, 4)$ of system $(9)$.

Let us think now of any collineation which replaces the four points $P_{1}$, $P_{2}, P_{3}, P_{4}$ by the set $P_{\alpha}, P_{\beta}, P_{\gamma}, P_{\delta}$ whose coordinates are respectively $\left(\alpha_{1}, \ldots, \alpha_{4}\right),\left(\beta_{1}, \ldots, \beta_{4}\right),\left(\gamma_{1}, \ldots, \gamma_{4}\right)$ and $\left(\delta_{1}, \ldots, \delta_{4}\right)$. The new set of points, in view of (28), determines a non-degenerate tetrahedron which by (29), (8) and (9) bears the same relation to a surface $\Sigma$ dualistic to $S$ as does the tetrahedron $P_{y} P_{z} P_{p} P_{\sigma}$ to $S$. That such a collineation will make to correspond to a fixed tetrahedron an $\infty^{1}$ of tetrahedrons is explained by the fact that the coefficients of the corresponding equations of transformation will be functions of the parameter $x$. We may now state the

THEOREм: Given a ruled surface $S$ generated by a one-parameter family of lines $L_{y z}$, and a determinant of the fourth order $\equiv 0$, whose elements are functions of the parameter of the surface S. If the elements in the columns of this determinant are the coordinates of the vertices of a tetrahedron which is fixed in space, then the elements in the rons are the coordinates of the vertices of a moving tetrahedron $T$, one edge $g$ of which generates a surface $\Sigma$ dualistic to $S$. Moreover four of the six edges of $T$ lie upon the quadric which osculates $\Sigma$ along $g$. 
The loci of the two points $P_{y}$ and $P_{z}$ of the generator $L_{y z}$ of $S$ are any two distinct curves of the surface. If in system (A) we have

$$
p_{11}=p_{22}=u_{12}=u_{21}=0 \text {, }
$$

then $P_{y}$ and $P_{z}$ are the flecnodes of $L_{y ; z}$ and their loci, $C_{y}$ and $C_{z}$, are the two branches of the flecnode curve of the surface $\left(^{*}\right)$. In view of $(30)$ and $(6)$, (A) becomes

$y^{\prime \prime}+p_{12} z^{\prime}+q_{11} y+{ }^{1} / 2 p_{12}^{\prime} z=0, \quad z^{\prime \prime}+p_{21} y^{\prime}+{ }^{1 / 2} p_{21}^{\prime} y+q_{22} z=0$.

With reference to the coordinate system already employed, the equations of the planes which osculate $C_{y}$ and $C_{z}$ at $P_{y}$ and $P_{z}$ are $\left(^{* * *}\right)$

$$
p_{12} x_{2}+p_{12}^{2} x_{3}-p_{12}^{\prime} x_{4}=0, \quad p_{21} x_{1}-p_{21}^{\prime} x_{3}+p_{21}^{2} x_{4}=0 \text {. }
$$

The coordinates of $\left(31_{1}\right)$ are

$$
k=0, \quad \lambda=p_{12} \omega, \quad \mu=p_{12}^{2} \omega, \quad \nu=-p_{12}^{\prime} \omega,
$$

where $\omega$ is an arbitrary function of $x$ not identically zero.

For system (F) conditions (10) become

$$
\left.\begin{array}{c}
2 k^{\prime}=n k-p_{12} \lambda+\mu, 2 \lambda^{\prime}=-p_{21} k+n \lambda+\nu \\
2 \mu^{\prime}=\left(p_{12} p_{21}-4 q_{11}\right) k+n \mu-p_{12} \nu, 2 v^{\prime}=\left(p_{12} p_{21}-4 q_{22}\right) \lambda-p_{21} \mu+n_{1} \nu .
\end{array}\right\}
$$

Substituting in (33) from (32) we find that $\left(33_{1}\right)$ is identically satisfied, that except for the factor $p_{12}\left({ }^{* * * *}\right),\left(33_{2}\right)$ and $\left(33_{8}\right)$ each becomes

while $\left(33_{4}\right)$ gives

$$
2 p_{12} \omega^{\prime}+3 p_{12}^{\prime} \omega-p_{12} n \omega=0
$$

$$
p_{12}^{\prime} \omega^{\prime}+\left(p_{22}^{\prime \prime}-2 p_{12} q_{22}\right) \omega-1 / 2 p_{12}^{\prime} n \omega=0 .
$$

Multiplying (34) by $p_{12}^{\prime}$ and (35) by $2 p_{12}$ and subtracting gives

$$
\left(4 p_{12}^{2} q_{22}-2 p_{19} p_{12}^{\prime \prime}+3 p_{12}^{2}\right) \omega=0 \text {. }
$$

(*) W., pp. 149,150 .

(**) (A) can be reduced to (F) only when the two branches of the flecnode curve are distinct.

(***) W., p. 214.

$\left(*^{* *}\right)$ If $p_{12}=0, C_{y}$ is a straight line and the osculating plane is indeterminate. We suppose here that $p_{12}=1=0$. 
In view of the arbitrary character of $\omega$ it results that

$$
4 \Delta_{1} \equiv 4 p_{12}^{2} q_{22}-2 p_{12} p_{12}^{\prime \prime}+3 p_{12}^{\prime_{2}}=0 \text {. }
$$

We conclude then that if the plane osculating the branch $C_{y}$ of the flecnode curve of $S$ at one of its points $P_{y}$ is to be independent of the parameter of the surface, that is, it $C_{y}$ is to be a plane curve, it is necessary that $\Delta_{1}=0\left(^{*}\right)$. A similar condition for the other branch $C_{z}$ of the flecnode curve of $S$ is

Now let

$$
4 \Delta_{2} \equiv 4 p_{21}^{2} q_{11}-2 p_{21} p_{21}^{\prime \prime}+3 p_{21}^{\prime \prime}=0
$$

$$
k x_{1}+\lambda x_{2}+\mu x_{3}+y x_{4}=0
$$

be a fixed plan and let us suppose that the two planes of (31) always intersect in a line of this plane, that is, that

$$
\begin{aligned}
k x_{1}+\lambda x_{2}+\mu x_{3}+\nu x_{4} \equiv \rho\left(p_{12} x_{2}+p_{12}^{2} x_{3}-p_{12}^{\prime} x_{4}\right)+ & \\
& +\psi\left(p_{21} x_{1}-p_{21}^{\prime} x_{3}+p_{21}^{2} x_{4}\right),
\end{aligned}
$$

where $\varphi, \psi$ are arbitrary functions distinct from zero. We have

$$
k=p_{21} \psi, \quad \lambda=p_{12} \varphi, \quad \mu=p_{12}^{2} \varphi-p_{21}^{\prime} \psi, \quad \nu=-p_{12}^{\prime} \varphi+p_{21}^{2} \psi
$$

Conditions (33) now become

$$
\begin{gathered}
2 p_{21} \psi^{\prime}+3 p_{21}^{\prime} \psi-p_{21} n \psi=0 \\
2 p_{12} \varphi^{\prime}+3 p_{12}^{\prime} \varphi-p_{12} n \varphi=0 \\
2 p_{12}^{2} \varphi^{\prime}-2 p_{21}^{\prime} \psi^{\prime}+3 p_{12} p_{12}^{\prime} \varphi-\left(2 p_{21}^{\prime \prime}-4 p_{21} q_{11}\right) \psi- \\
-p_{12}^{2} n \varphi+p_{21}^{\prime} n \psi=0 \\
-2 p_{12}^{\prime} \varphi^{\prime}+2 p_{21}^{2} \psi^{\prime}-\left(2 p_{12}^{\prime \prime}-4 p_{12} q_{22}\right) \varphi+3 p_{21} p_{21}^{\prime} \psi+ \\
+p_{12}^{\prime} n \varphi-p_{21}^{2} n \psi=0
\end{gathered}
$$

The determinant of the coefficients of $\varphi^{\prime}, \psi^{\prime}, \varphi, \psi$ in these equations is, except for the faclor $2^{6} p_{12} p_{21}, \Delta_{1} \Delta_{2}$ so that we must have either $\Delta_{1}=0$ or $\Delta_{2}=0$, that is, one or other branch of the flecnode curve must be plane. And this is evidently sufticient. We therefore state the

THEORвм: In order that the line of intersection of the planes osculating

(*) It is also sufficient. See a paper by the author in the Transactions, Oct. 1915. 
the flecnode curve of a given ruled surface at the two points in which it is cut by a generator of the surface may envelope a plane curve, it is necessary and sufficient that one branch of the flecnode curve be a plane curve (*).

Let $l$, with coordinates $\left(\omega_{12}, \omega_{13}, \omega_{14}, \omega_{23}, \omega_{42}, \omega_{34}\right)$ be a fixed line. The coordinates of the planes $l P_{y}$ and $l P_{z}$ determined by this line and the two points $P_{y}(1,0,0,0)$ and $P_{z}(0,1,0,0)$ are by $(17)$

$$
\begin{array}{ll}
k_{y}=0, & \lambda_{y}=-\omega_{34}, \quad u_{y}=-\omega_{42}, \quad \nu_{y}=-\omega_{25}, \\
k_{z}=\omega_{34}, & \lambda_{z}=0, \quad \mu_{z}=-\omega_{14}, \quad \nu_{z}=\omega_{13} .
\end{array}
$$

If we employ system (F), that is, use the two branches of the flecnode curve of $S$ for directrix curves, then the point-plane correspondence determined by the linear complex which osculates $S$ along $L_{y z}$ is given by the equations $(* *)$

$$
k=p_{12} \gamma, \quad \lambda=-p_{21} \delta, \quad \mu=-p_{12} \alpha, \quad \nu=p_{21} \beta .
$$

By means of (41) we find that the points corresponding to the planes $l P_{y}$ and $l P_{z}$ have respectively the coordinates

$$
\begin{aligned}
& \alpha_{1 y}=\frac{\omega_{42}}{p_{12}}, \quad \beta_{y}=-\frac{\omega_{28}}{p_{21}}, \quad \gamma_{y}=0, \quad \delta_{y}=\frac{\omega_{34}}{p_{21}}, \\
& \alpha_{z}=\frac{\omega_{14}}{p_{12}}, \quad \beta_{z}=\frac{\omega_{19}}{p_{21}}, \quad \gamma_{z}=\frac{\omega_{34}}{p_{12}}, \quad \delta_{z}=0,
\end{aligned}
$$

and from these we obtain the coordinates of the line $l^{\prime}$ determined by them, namely the polar reciprocal of the given line.l with respect to the osculating linear complex. They are

$$
\left.\begin{array}{lll}
0_{12}=p_{12} p_{21} \omega_{12} \varphi, & 0_{13}=-p_{21}^{2} \omega_{42} \varphi, & 0_{14}=p_{12} p_{21} \omega_{14} \varphi, \\
0_{23}=p_{12} p_{21} \omega_{23} \varphi, & 0_{42}=-p_{24}^{2} \omega_{13} \varphi, & 0_{34}=p_{12} p_{21} \omega_{34} \varphi,
\end{array}\right\}
$$

where $\varphi$ is an arbitrary function $\equiv=0$. Now for system (F) equations (16)

${ }^{*}$ ) It is assumed here that the two branches of the flecnode curve are distinct else a form of system (A) different from (F) would be necessary.

(**) W., p. 206. 
take the form

$$
\begin{gathered}
2 \omega_{12}^{\prime}=\zeta \omega_{12}-u_{22} \omega_{14}+u_{11} \omega_{23}, \quad 2 \omega_{13}^{\prime}=\zeta \omega_{13}+p_{21}\left(\omega_{14}+\omega_{23}\right), \\
2 \omega_{14}^{\prime}=-\omega_{12}+p_{12} \omega_{13}+\zeta \omega_{14}-p_{21} \omega_{42}-u_{11} \omega_{34}, \\
2 \omega_{23}^{\prime}=\omega_{12}+p_{12} \omega_{13}+\zeta \omega_{23}-p_{21} \omega_{42}+u_{22} \omega_{34}, \\
2 \omega_{42}^{\prime}=-p_{12}\left(\omega_{14}+\omega_{23}\right)+\zeta \omega_{42}, \quad 2 \omega_{34}^{\prime}=-\omega_{14}+\omega_{23}+\zeta \omega_{34} .
\end{gathered}
$$

We have from (42) by making use of (43)

$$
\begin{aligned}
& 20_{12}^{\prime}=\left[\xi+4\left(q_{12} / p_{12}+q_{21} / p_{21}\right)\right] 0_{12}-u_{22} 0_{14}+u_{11} 0_{34}, \\
& 20_{13}^{\prime}=\left[\xi+8 q_{21} / p_{21}\right] 0_{13}+p_{21}\left(0_{14}+0_{23}\right), \\
& 20_{14}^{\prime}=-0_{12}+p_{12} 0_{13}+\left[\xi+4\left(q_{12} / p_{12}+q_{21} / p_{22}\right)\right] 0_{14}-p_{21} 0_{42}-u_{11} 0_{34}, \\
& 20_{23}^{\prime}=0_{12}+p_{12} 0_{18}+\left[\xi+4\left(q_{12} / p_{12}+q_{21} / p_{21}\right)\right] 0_{23}-p_{21} 0_{42}+u_{22} 0_{34}, \\
& 20_{42}^{\prime}=-p_{12}\left(0_{14}+0_{23}\right)+\left[\xi+8 q_{13} / p_{12}^{\prime} \theta_{42},\right. \\
& 20_{34}^{\prime}=-0_{14}+0_{23}+\left[\xi+4\left(q_{12} / p_{12}+q_{11} / p_{21}\right)\right] 0_{34},
\end{aligned}
$$

where $\xi=\zeta+\left.2 \varphi^{\prime}\right|_{\varphi}$. Comparison of (43) and (44) shows that the line $l^{\prime}$ will be fixed in space if and only if

that is if

$$
q_{12} / p_{12}+\left.q_{21}\right|_{p_{21}}=2 q_{12} / p_{12}=\left.2 q_{21}\right|_{p_{21}}
$$

$$
p_{12} q_{21}-p_{21} q_{12}=0
$$

Now $p_{12} q_{21}-p_{21} q_{12}$ is a factor of the invariant $\theta_{9}$ of system (F) (*) and WilczYnski has shown that if $\theta_{9}=0$, the ruled surface $S$ belongs to a linear complex (**). On the other hand if $S$ does belong to a linear complex, all the linear complexes osculating $S$ along. its generators coincide with the complex to which $S$ belongs and hence if $l$ is fixed so is $l$. We may then state the

THEOREM: In order that the polar reciprocals of a line fixed in space taken with respect to the linear complexes which osculate a ruled surface $S$ along its generators, shall coincide, in a second fixed line, it is necessary and sufficient that $s$ belong to a linear complex.

If one takes the polar reciprocals of a fixed line with respect to the quadrics which osculate $S$ along its generators it is easily shown that these can coincide in a second fixed line if, and only if, $S$ is itself a quadric.

(*) W., p. 119 .

(**) W., p. 170 . 
With respect to the centers of the quadrics osculating a ruled surface, Demoulin (*) has proved a theorem which may be stated thus: let $C$ be the center of the quadric which osculates a ruled surface $S$ along a generator $g$. The tangent at $C$ to the locus of $C$ cuts $g$ in a point midway between the flecnodes of $g$. We proceed to prove a theorem of which the above is a special case.

Let

$$
k x_{1}+\lambda x_{2}+\mu x_{3}+\nu x_{4}=0,
$$

be the equation of a fixed plane. $\Pi$. The pole $P$ of this plane with respect to the quadric $x_{1} x_{4}-x_{2} x_{3}=0$ osculating $S$ along a generator $L_{y z}$ has coordinates $(\nu,-\mu,-\lambda, k)$ and its expression is

or, by (1),

$$
p=v y-\mu \cdot z-\lambda p+k \tau,
$$

$$
p=\left(p_{21} k-p_{11} \lambda+v\right) y+\left(p_{22} k-p_{12} \lambda-\mu\right) z-2 \lambda y^{\prime}+2 k z^{\prime} .
$$

By making use of (A)

$$
\left.\begin{array}{rl}
p^{\prime}=\left[p_{21} k^{\prime}-p_{11} \lambda^{\prime}+v^{\prime}+\left(p_{21}^{\prime}-2 q_{21}\right) k+\left(2 q_{11}-p_{11}^{\prime}\right) \lambda^{\prime} y+\right. \\
\quad+\left[p_{22} k^{\prime}-p_{12} \lambda^{\prime}-\mu^{\prime}+\left(p_{22}^{\prime}-2 q_{22}\right) k+\left(2 q_{12}-p_{12}^{\prime}\right) \lambda\right] z+ \\
+\left[-2 \lambda^{\prime}-p_{21} k+p_{11} \lambda+v\right] y^{\prime}+\left[2 k^{\prime}-p_{22} k+p_{12} \lambda-\mu\right] z^{\prime} .
\end{array}\right\}
$$

As the parameter $x$ varies the point $P$ generates a space curve. We write the expression for the general point on the tangent to this curve in the form $\theta p+\varphi p^{\prime}$ where $\theta$ and $\varphi$ are arbitrary functions of $x$ not simultaneously zero. We have from (47) and (48)

$$
\begin{aligned}
\theta p+\varphi p^{\prime}=\left(\theta \left(p_{21} k\right.\right. & \left.-p_{11} \lambda+\nu\right)+\varphi\left[p_{21} k^{\prime}-p_{11} \lambda^{\prime}+\nu^{\prime}+\right. \\
& \left.+\left(p_{21}^{\prime}-2 q_{21}\right) k+\left(2 q_{11}-p_{11}^{\prime}\right) \lambda\right] \mid y+ \\
& +i \theta\left(p_{22} k-p_{12} \lambda-\mu\right)+\varphi\left[p_{22} k^{\prime}-p_{12} \lambda^{\prime}-\mu^{\prime}+\right. \\
& \left.\left.+\left(p_{22}^{\prime}-2 q_{22}\right) k+\left(2 q_{12}-p_{12}^{\prime}\right) \lambda\right]\right\} z+ \\
& \left.+i-2 \lambda \theta+\left(-2 \lambda^{\prime}-p_{21} k+p_{11} \lambda+\nu\right) \varphi\right) y^{\prime}+ \\
& +\left(2 k \theta+\left(2 k^{\prime}-p_{22} k+p_{12} \lambda-\mu\right) \varphi\right) z^{\prime}
\end{aligned}
$$

Equating to zero the coefficients of $y^{\prime}$ and $z^{\prime}$ in (49) and solving for

(*) Comptes Rendus, 1908, June, p. 1381. 
the ratio $\theta / \%$ we have

$$
{ }^{\theta} / p=\frac{-2 \lambda^{\prime}-p_{21} k+p_{11} \lambda+v}{2 \lambda}=\frac{-2 k^{\prime}+p_{22} k-p_{12} \lambda+\mu}{2 k},
$$

and each of these values, in view of $(10)$, reduces to $-1 / 2\left(n+p_{11}+p_{22}\right)$. Using this value and again employing (10) to remove $k^{\prime}, \lambda^{\prime}, \mu^{\prime}, v^{\prime},(49)$ reduces to

$$
\left.\begin{array}{rl}
-\left(n+p_{11}+p_{22}\right) p+2 p^{\prime}=\left[2 u_{21} k\right. & \left.\left.+\left(u_{22}-u_{11}\right)\right)\right] y+ \\
& +\left[\left(u_{22}-u_{11}\right) k-2 u_{12} \lambda\right] z .
\end{array}\right\}
$$

The points $P$ are in one-to-one correspondence with the generators $L_{y z}$ of $S$ and equation (50) shows that the tangent at $P$ to the locus of $P$ intersects that generator of $S$ to which $P$ corresponds. If now we use for directrix curves the two branches of the flecnode curve of $\mathcal{S}$, employing system (F) in place of system (A), equation (50) reduces to

$$
-n p+2 p^{\prime}=\left(u_{22}-u_{11}\right)(\lambda y+k z),
$$

so that the expression for the point of intersection of $L_{y z}$ with the corresponding tangent to the locus of $P$ is, omitting the factor $\left(u_{22}-u_{11}\right)$,

$$
\lambda y+k z .
$$

But the point in which the plane $\Pi$ cuts $L_{y z}$ has for its expression

$$
\lambda y-k z \text {. }
$$

These two points are evidently harmonic conjugates with respect to the two points $P_{y}$ and $P_{z}$ so that we have the

THeовем: If $P$ is the pole, with respect to the quadric which osculates a ruled surface $S$ along a generator $g$, of an arbitrary fixed plane $\Pi$ which cuts $g$ in a point $G$, then the tangent at $P$ to the locus of $P$ cuts $g$ in a point which is harmonically separaled from $G$ by the flecnodes of $g$.

In order to obtain Demoulin's theorem quoted above it is only necessary to take $I I$ as the plane at infinity.

By (41) the coordinates of the point $Q$ of $\Pi$ which corresponds to $\Pi$ by means of the linear complex which osculates $S$ along $L_{y z}$, are

$$
\alpha=-p_{21} \mu, \quad \beta=p_{12} \vee, \quad \gamma=p_{21} k, \quad \delta=-p_{12} \lambda,
$$


and its expression is

$$
q=-p_{21 ; j} y+p_{12} v z+p_{21} k p-p_{12} \lambda \sigma .
$$

By making use of (F), (1) and (33), we find that

$$
\begin{aligned}
2 q^{\prime}=-\left(4 q_{21}+p_{21} n\right) \mu y+( & \left(4 q_{12}+p_{12} n\right) v z+ \\
& +\left(4 q_{21}+p_{21} n\right) k p-\left(4 q_{12}+p_{12} n\right) \lambda \sigma .
\end{aligned}
$$

The general point on the tangent at $Q$ to the locus of $Q$ will have for its expression

where

$$
2\left({ }^{\natural} q+\varphi q^{\prime}\right)=-A \mu y+B \nu z+A k \rho-B \lambda \sigma,
$$

$$
A=2 p_{21} \theta+\left(4 q_{21}+p_{21} n\right) \varphi, \quad B=2 p_{12} \theta+\left(4 q_{12}+p_{12} n\right) \varphi,
$$

and $\theta$ and $\varphi$ are arbitrary functions not simultaneously zero. Since $\theta$ and $\varphi$ can be so chosen that either $A=0$ or $B=0$ it results that this tangent line intersects both the line $P_{y} P_{p}$ and the line $P_{z} P_{a}$. But these two lines are the flecnode tangents of the generator $L_{y z}$. We therefore state the

Theorem: If $\mathrm{\Pi}$ is a plane fixed in space then the lines of $\mathrm{\Pi}$ determined by the points of intersection. of II with corresponding pairs of flecnode tangents of a ruled surface $S$ envelope a curve which is at the same time the locus of the points which correspond to $\mathrm{I}$ by means of the osculating linear complexes of $S$.

The proofs of the foregoing theorems serve to indicate the adaptability of the conditions obtained in part I and it seems reasonable to suppose that these equations of condition can be applied extensively in further development of the projective theory of ruled surfaces.

The University of Washington,

March 1, 1916. 